\title{
Astronomy Development in Morocco: a Challenge to Stimulate Science and Education
}

\author{
Khalil Chamcham \\ King Hassan II University - Ain Chock, Faulty of Science, B.P.5366, \\ Maarif, Casablanca, Morocco.e-mail: chamcham@star.cpes.susx.ac.uk
}

\begin{abstract}
From my experience in Morocco, I discuss the difficulties one can face while trying to set up projects in a country where astronomy is a forgotten science: everything has to be built from scratch and, at the same time, one is required to keep up the pace at the international level. But, on the other side, it is quite a relief to see the strong demand from students and the public. In these circumstances even professional astronomy cannot survive without feedback from the public and longterm investment in education at all levels.
\end{abstract}

\section{Introduction:}

In the early 1980 s the Moroccan government officially encouraged the creation of an astronomy unit at the CNR, the Moroccan national institution coordinating scientific research. At that time, a small group of people trained in France was involved in setting up a network for solar oscillations, IRIS. One of the tasks of this group was to coordinate astronomy nationwide, in order to create other units in universities and engineering schools, and facilities were consequently provided to achieve this goal. Researchers from the university of Casablanca, coming from different areas in physics, established contacts to work in this context, but very soon the relationship between the coordinating group and its mother institution became very confrontational and the unit was diluted.

Also, from the beginning, the IAU expressed its support for Moroccan astronomy and Professor Jorge Sahade, acting as the IAU president, made an official visit to Morocco to express to the Moroccan authorities the support of the IAU for astronomy development. His visit was followed by the organization of an ISYA, in 1991, in Morocco, which was a very successful school. At the same time Japanese officials expressed their willingness to support Morocco in the development of astronomical facilities (i.e. a planetarium and an observatory).

This was a great period when Moroccan astronomy could have gone very far with this local and international encouragement had not some individuals in administrative positions failed to see the opportunity to invest in science and in the national coordination of astronomers scattered around the country. Unfortunately Moroccan astronomers lost this chance to organize themselves and to develop national facilities. From then on the above mentioned conflicts became a justification for unpleasant harassements. 


\section{Present-Day Situation of Astronomy in Morocco}

Despite this negative picture, some individuals carried on their intiatives and developed activities or units as part of the phyiscs department of their institution. These activites have been developed in the following places:

\subsection{Rabat}

- At the CNR, two people carried on working on tomography and solar oscillations.

- One person moved from the CNR to the University of Rabat, but did not develop any activity. However he is mainly involved in amateur activities as part of a cultural association.

- The United Nations has designated the engineering school, EMI, as the home of the UN space Centre in Africa and a satellite project. This Centre is operational in principle, but it is not open to other people of the school. Also, the Moroccan Ministry of Research and Higher Education has initiated the creation of a national network for space research (RUSTE) wich is based at the same school. The activities of this network have been limited to local meetings and popularisation.

- Public Astronomy: a cultural association has built a small observatory to house a 50-cm telescope donated by the French government and a small planetarium. These facilities are in principle open to the public.

\subsection{Marrakesh}

Another initiative was carried on by an individual at the University of Marrakesh, previously involved in the solar-oscillation project IRIS. He could include a course in astronomy as part of the high-energy physics group in the University. Also, he developed some amateur activity in the city of Marrakesh.

\subsection{Oujda}

A couple of physicists have been working on astro-particles in collaboration with Italian groups. They have been active in developing research projects in connection with high-energy astrophysics and recently they submitted an M.Sc. project to the Moroccan authorities.

\subsection{Casablanca:}

Since 1988, local astronomy meetings have organised at the University of Casablanca, as part of the activities of the nuclear and theoretical physics group and with the collaboration of French astrophysicists. These activities have been a stimulus, motivating students to come to astronomy and generating a gradual need to include astronomy as part of the physics curriculum. In fact, an undergraduate course and a postgraduate course have been developed and some of the students are fairly well advanced in their work.

There is now an Astronomy and Astrophysics Group structured as follows

- Two full professors, 
- five Ph.D. students: one is now at SISSA and four are working between Casablanca and Italy with grants offered by Roma Observatory and the University of Pisa.

- Six postgraduate and seven undergraduate students.

- The curriculum focuses on fundamental astronomy, astrodynamics, stellar structure and evolution, galaxy formation, cosmology, numerical methods and English (i.e. the postgraduate course is attended by non-astronomy students from plasma physics, theoretical and particle physics).

- Research projects of the Group are concerned with celestial mechanics, CCD photometry, solar physics, stellar evolution, galaxy evolution and cosmology.

- The facilities available to the Group are: 1 PC, the IAU traveling telescope $+\mathrm{CCD}$, the Edinburgh teaching package, Hands On Astrophysics and a collection of textbooks and proceedings. Most of this equipement has been acquired thanks to the support of the IAU, the UN Office for Outer Space Affairs, and a contribution from our faculty.

\section{The Public Demand}

The administration of academic institutions in Morocco is heavily bureaucratic and often appears to work for its own sake, as if the administrators have forgotten that they were nominated to serve the interest of students and staff. It sometimes seems as if science itself is looked upon as a marginal activity and that asking for budgeted money to be spent on the needs of students or the support of research is seen as a threat to the system, to be countered by personal harassment. These attitudes influence even some of the academics so that a small group trying to modernize teaching and research needs very strong international support. This is the situation of astonomy in Morocco and each step forward has been a real struggle.

Security is constant concern in our society and this also affects astronomy. For example, access to the Internet, seen as a source of subversive information, is strictly controlled. A telescope may be viewed as a device that can see far into a city (rather than deep into the sky) and take pictures: it may be prohibited for security reasons, although this may not be said explicitly.

Where science and culture cannot make progress, the irrational grows rapidly. On the other hand, people struggle for survival and want a good education, either for themselves or at least for their children. They want that education to include modern science and what the media show about the results of space research and other new discoveries in the developed world. From this perspective, astronomy benefits from wide public support and if, the administration has recognised the importance of astronomy, it is thanks to public pressure and the demand for astronomical activities from the students themselves. 


\section{The Role of the IAU and International Collaboration}

In the case of Morocco, the breakthrough came from the investigation of Alan Batten when he visited the country on behalf of the IAU. His understanding of the situation with all its ups and downs helped not only to give confidence to the astronomy community but also to prepare the administration to a more open position toward it.

Batten's visit allowed the preparation of a TAD contract between the IAU and the University of Casablanca, which was finalized during a visit by Donat Wentzel and Johannes Andersen. This had a great positive impact, moderating the attitude of the administration toward the astronomy community and establishing self-confidence and dynamism amongst students and the astronomers themselves. This can be understood if one knows that students who wished tc follow the undergraduate astronomy course were pressured, or news was spread from unknown sources that the astronomy course was cancelled.

The other initiative which backed up astronomy teaching and research in the University of Casablanca is the kind support received from the Italian Astronomical Society (SAIt) and the Universites of Sussex and Glasgow. The SAIt provided travel grants and fellowships to students and staff members whereas the latter offered remote computing facilities and visiting grants. Particularly, the Osservatorio Astronomico di Roma has been very supportive in this respect and Pisa University is now donating computing facilities and providing training courses to all students. The Osservatorio Astronomico di Trieste has also been supporting research projects and the training of students. Thanks to these collaborations, we could set up research projects and strengthen our curriculum.

\section{The Importamce of Computing}

Our teaching programmes are out of date and lack any sense of creativity. The undergraduate science programme is still based on the physics and mathematics of the early twentieth century and, in most advanced-level courses dates back to the early 1950s. When one sees that the table of contents of our official curriculum is nothing but a photocopy of the tables of contents of French textbooks, one can understand the effort involved to think of what kind of science we need to catch up with modern development and what kind of citizens we want to produce.

Considering these facts and what I have said above, it became clear to our Astronomy and Astrophysics Group that investment in computing is fundamental to back up students' projects and research. Also, in the absence of access to library facilities and journals, the Internet is now playing a major role in education and research by allowing people to access most of the necessary information they look for. It is essentially a way to introduce students to modern science without the need to go through the official channels, which in any case will never respond to any suggested reform of the curricula (modifying or replacing one single chapter of a course may require going through all the official institutions of the country, which is deterring).

The good thing about astronomy is that it is a science which fascinates people and excites their imaginations. This allows one to build strong relationships 
with people from all generations and social backgrounds. With a few pictures and some hand-waving, one can explain complex physics to "gran'ma" and even justify the technological and economical impact of astronomy on society, not excluding the cultural feedback.

In the case of Morocco, astronomy showed that even educated scientists who abandoned any hope of carrying on their scientific career could discover some joy by becoming interested in astronomy. The experience with the public also showed that people, at the end of a lecture or an open day, discover that the study of the sky provides a way to modernity and higher education, as long as they have the right to have a sky above their head.

\section{Conclusion}

It is exciting to carry this endeavour of bringing new hope and new light to individuals and a whole country through the mother of sciences, astronomy. I found it necessary to describe the social and ideological environment where astronomy used to be an honorable science, but is nowadays struggling for survival. This experience shows that societies do not always aim for the best, but a minority of individuals can aim for it and take the lead. The most positive aspect of this experience is the strong and permanent support amongst the astronomical community without which some astronomers could not survive in their own country. But wherever we go it is thanks to the taxpayers that things can change and improve, not only because of their money, but by their strong voice expressing what they want for their lives. I am glad that astronomy is teaching us that science is not isolated from the rest of our human experience.

\section{Acknowledgment}

I acknowledge the IAU financial support to attend the GA 2000 in Manchester and for the IAU-TAD programme in Morocco. I am very grateful for the permanent support of Alan Batten, Donat Wentzel, Derek McNally, John Percy and Hans Haubold. The AAVSO is kindly providing educational material.

\section{Discussion}

Hearnshaw noted that in many Moslem countries, particularly Malaysia, astronomers have successfully gained funds by using the link between Islam and astronomy. He wondered why Chamcham was reluctant to do this. Chamcham replied that he was aware of the Malaysian experience and had himself doe some work on Islamic astronomy. Funding applications to Moslem institutions, however, were compicated by bureaucratic procedures and often unsuccessful.

Chambliss asked which of Arabic, French or English was the language of science instruction in Morocco. Chamcham replied that the official language at high-school and college levels is Arabic and at university level it is French. Only a few people at his university spoke English, although he taught it as part of the postgraduate physics and required mature students who came to astronomy to take private tuition in English. 
da Costa suggested that colleagues in Spain and Portugal could assist Moroccan astronomers. Chamcham replied that he had a few contacts with Spanish colleagues and IAU representatives had suggested that he send students to Spain for training in observational techniques. He had met Portugueses students in the U.K. but otherwise knew only da Costa himself. He would be happy to base future collabotration on these relationships. 